\title{
Fourth International Conference on High-Temperature Capillarity 2004 (HTC-2004) to Convene in Italy
}

The Fourth International Conference on High-Temperature Capillarity 2004 (HTC2004) will be held in Sanremo, Italy, on March 31-April 3, 2004. Special sessions are also being offered in Genova, Italy, as part of the "Genova, European Capital of Culture, 2004" celebrations. The meeting is organized by the Institute for Energetics and Interphases of the Italian National Research Council (IENI-CNR). Under the guidance of program chairs Nicolas Eustathopoulos (Institute National Polytechnique de Grenoble, CNRS, France) and Alberto Passerone (IENI-CNR, Italy), HTC-2004 will present the latest scientific advancements and developments in the field of high-temperature capillarity (energetic properties of surfaces and interfaces and their effects on high temperature materials processing).

Capillary phenomena are significant in many aspects of daily life and engineering. In the science and engineering of materials, high-temperature capillary phenomena are particularly important for their role in the processing and behavior of many materials. HTC-2004 will provide a forum for scientists from academia and industry to present and discuss scientific advances in their research on this topic, including results of experiments, theoretical models, new experimental techniques, and new processes.

The scientific program will consist of plenary sessions, introduced by invited speakers, and poster sessions, which will be held every day. The central focus of this conference is surfaces and interfaces, wetting, and capillarity in materials processing. Relevant subtopics include liquid surfaces of metals, glasses, and salts; metal-metal, metal-ceramic, metal-glass, and ceramic-ceramic interfaces; capillarity in crystal growth, grain growth, and solidification; capillarity in microgravity; Marangoni phenomena; corrosion and embrittlement by liquid metals; electrocapillarity and associated phenomena; new experimental methods; modeling and simulation; foundry processes and metallurgical production; processing of composite materials; and joining.

Abstract deadline is July 31, 2003.

HTC-2004 is the fourth in a series on high-temperature capillarity. Previous conferences were held in Smolenice, Slovakia, in 1994; Krakow, Poland, in 1997; and Kurashiki, Japan, in 2000. This year, the conference is endorsed by CNR, the Materials Research Society, the Joining and Welding Research Institute at Osaka University (NEDO International Joint Research Grant 01MB7, K. Nogi's Team), Center of Excellence for Solidification of Undercooled Melts-ZEUS, European Space Agency, Istituto Italiano della Saldatura, CONFAPI, and Azienda di Promozione Turistica Riviera dei Fiori.

For information on the conference or to register, contact A. Passerone, HTC2004 organizing committee coordinator, tel. 39-010-6475716, fax 39-010-6475700, or e-mail htc4@ge.ieni.cnr.it; or access the conference Web site www.ieni.cnr.it/ conferences.htm. 